\title{
A NOTE ON A FIXED POINT THEOREM OF OKHEZIN
}

\author{
A. TOMINAGA
}

(Communicated by Dennis K. Burke)

\begin{abstract}
In 1985 V. P. Okhezin proved that the cartesian product of a $B$ space $X$ and a compact metric AR space has the fixed point property. In this paper it is shown that the cone over $X$ and the suspension of $X$ have the fixed point property.
\end{abstract}

\section{INTRODUCTION}

A nest is a monotone, increasing sequence of sets. A $B$-space $X$ is an arcwise connected $T_{2}$-space such that every nest of arcs of $X$ is contained in an arc of $X$. A space $S$ has the fixed point property (abbreviated FPP) if for every continuous mapping $f$ of $S$ into itself, there exists a point $p$ of $S$ such that $f(p)=p$.

In 1946 Young [9] proved that every $B$-space has FPP. In 1954 Borsuk [1] showed that every arcwise connected, hereditarily unicoherent continuum $X$ is a $B$-space and proved that $X$ has FPP by a different method than Young's. In 1969 Holsztyński [3] generalized the above Young's result by a method similar to Borsuk's method. The" $B$-space" named by Holsztyński derives from BorsukYoung arcwise connected space.

In 1985 Okhezin [5] verified that the cartesian product of a $B$-space and a compact metric AR space has FPP. In this note we shall prove the following theorem by his method.

Theorem. Let $X$ be a $B$-space and $Y$ a compact metric AR space. Let $F$ be a closed subset of $Y$, which may be empty. Define an equivalence relation $\sim$ in the cartesian product $X \times Y$ by $(x, y) \sim\left(x^{\prime}, y\right)$ for every $y \in F$ and any $x$, $x^{\prime} \in X$. Then the quotient space $Q(X)=(X \times Y) / \sim$ has FPP.

Corollary 1 [5]. The cartesian product of a B-space and a compact metric $A R$ space has FPP.

Corollary 2. The cone over a B-space has FPP.

Corollary 3. The suspension of a B-space has FPP.

Received by the editors June 16, 1990.

1980 Mathematics Subject Classification (1985 Revision). Primary 54F20, 54H25.

$K e y$ words and phrases. $B$-space, fixed point property, cone, suspension. 


\section{Preliminary lemmas}

From the definition of $B$-space we can easily get

Lemma 1. $A$ B-space $X$ is uniquely arcwise connected, that is, for any two points $x, y$ of $X$ there is a unique arc in $X$ with end points $x, y$.

Definition 1 [8]. Let $X$ be a connected $T_{1}$-space. A nonempty subset $T$ of $X$ is an $A$-set if $X \backslash T$ is the union of a collection $\{C\}$ of open sets each having a single point $q \in T$ as its boundary

$$
X \backslash T=\bigcup C, \quad \text { Bd } C=q \in T .
$$

If $X$ is locally connected, then we may take all components of $X \backslash T$ as $\{C\}$.

Lemma 2. Let $X$ be a locally arcwise connected B-space. If $T$ is an arcwise connected, closed subset of $X$ then it is an A-set.

Proof. Since $T$ is closed and $X$ is locally connected, every component $C$ of $X \backslash T$ is open. Then $\mathrm{Bd} C$ consists of only one point. For, on the contrary, let $x_{1}, x_{2}$ be distinct points of $\mathrm{Bd} C$. Since $X$ is Hausdorff, there exist disjoint neighborhoods $U_{i}$ of $x_{i} \quad(i=1,2)$. Then a point $y_{i} \in U_{i} \cap C$ can be joined to $x_{i}$ by an arc $\alpha_{i}$ in $U_{i}$. Let $\beta$ be an arc in $T$ joining $x_{1}$ to $x_{2}$ and $\gamma$ an arc in $C$ joining $y_{1}$ to $y_{2}$. Then $\alpha_{1} \cup \beta \cup \alpha_{2} \cup \gamma$ contains a simple closed curve, contrary to Lemma 1.

Definition 2. Let $T$ be an $A$-set of a connected $T_{1}$-space $X$. Then define a function $r: X \rightarrow T$ by

$$
r(x)= \begin{cases}x & x \in T, \\ q=\operatorname{Bd} C & x \in C .\end{cases}
$$

If $X$ is locally connected, then $r$ is a continuous mapping [8] called the canonical retraction of $X$ onto $T$.

We easily have

Lemma 3. Let $X$ be a locally connected B-space, $T$ an $A$-set of $X$, and $r: X \rightarrow T$ the canonical retraction of $X$ onto $T$. Define $R: Q(X) \rightarrow Q(T)$ by $R(\langle x, y\rangle)=\langle r(x), y\rangle$, where $\langle x, y\rangle$ is the equivalence class of $(x, y) \in X \times Y$. Then $R$ is a retraction of $Q(X)$ onto $Q(T)$, called the retraction associated with $r$.

Definition 3. A countable fan is homeomorphic to the union of the closed line segments in the Euclidean plane $R^{2}$ joining the origin $(0,0)$ to the point $(1,1 / n)(n=1,2, \ldots)$. A countable comb is homeomorphic to the subspace $C_{0}$ of $R^{2}$ constructed as follows: Erect at $(1 / n, 0)(n=1,2, \ldots)$ in $R^{2}$ a perpendicular interval of length one. Then $C_{0}$ is the union of the erected intervals and the unit interval with end points $(0,0),(1,0)$.

Lemma 4 (cf. [4, p. 84]). Let $X$ be $a$ B-space and $T$ an arcwise connected subset of $X$ with infinitely many end points. Then $T$ contains a countable fan or a countable comb.

Proof. The arc in $X$ with end points $x, y$ is denoted by $[x, y]$. Define $(x, y)=[x, y] \backslash\{x, y\}$. Now pick a base point $a_{1} \in T$. According to [4] we first introduce an order $\leqq$ in $T$ by the rule: $x \leqq y$ if and only if $x \in\left[a_{1}, y\right]$. 
For $x \in T, M(x)=\{y \in T \mid x \leqq y\}$ and $L(x)=\{y \in T \mid y \leqq x\}$. The notation $x \wedge y$ means the point $\sup \{L(x) \cap L(y)\}$. A branch $B$ at $x$ is a subset of $M(x)$ that is maximal with respect to the property: If $y, z \in B \backslash\{x\}$ then $y \wedge z \in B \backslash\{x\}$. Note that every maximal element of $T$ is an end point of $T$ and the only other possible end point of $T$ is $a_{1}$.

Assume that $T$ contains neither countable fan nor countable comb. Then there are only finitely many branches at $a_{1}$. For if not, there would be a countable fan in $T$. Hence a branch $B_{1}$ at $a_{1}$ has infinitely many end points. Let $m_{1}$ be one of these end points. The arc $\left[a_{1}, m_{1}\right]$ contains only finitely many branch points. For if not, there would exist a countable comb.

Inductively we can construct three sequences $\left\{m_{i}\right\},\left\{a_{i}\right\}$, and $\left\{B_{i}\right\}$ with the following properties:

(i) $B_{i}$ is a branch at the point $a_{i}$ with infinitely many end points.

(ii) $m_{i}$ is an end point of $B_{i}$.

(iii) $a_{i+1} \in\left(a_{i}, m_{i}\right)$.

Denote the arc $\bigcup_{i=2}^{n}\left[a_{i}, a_{i+1}\right]$ by $A_{n}(n \geqq 2)$. Since $X$ is a $B$-space, the nest $\left\{A_{n}: n \geqq 1\right\}$ of arcs is contained in an arc of $X$. Let $\lim a_{i}=a$. Then the set $\left(\bigcup_{n=2}^{\infty} A_{n}\right) \cup\left(\bigcup_{i=1}^{\infty}\left[a_{i+1}, m_{i}\right]\right) \cup\{a\}$ is a countable comb, which is a contradiction. Thus $X$ contains a countable fan or a countable comb.

Lemma 5. Let $X, Y$, and $F$ be as in Theorem. Let $T$ be a finite tree in $X$ and define $Q(T)$ in the same way as $Q(X)$. Then $Q(T)$ has FPP.

Proof. If $F=Y$ then $Q(T)$ is homeomorphic to $Y$, and hence $Q(T)$ has FPP. If $F=\phi$ then $Q(T)=T \times Y$, and hence $Q(T)$ is a compact metric AR space. Therefore $Q(T)$ has FPP.

For the other case, suppose that $T$ is contained in the unit disk $D$ of the Euclidean plane. Let $\psi$ be a retraction of $D$ onto $T$. We may assume that $\max d(y, F) \leqq 1$ for $y \in Y$, where $d$ is a metric on $Y$. Then the set $H=$ $\{(d(y, F) \cdot x, y)(x, y) \in D \times Y\}$ is homeomorphic to $Q(D)$, which is defined in the same way as $Q(X)$, and the subset $K=\{(d(y, F) \cdot x, y)(x, y) \in T \times Y\}$ of $H$ is homeomorphic to $Q(T)$. Obviously $H$ is a retract of $D \times Y$. Since $D \times Y$ has FPP, so does $H$. Furthermore a retraction $g: H \rightarrow K$ is defined by

$$
g((x, y))= \begin{cases}(d(y, F) \cdot \psi(x / d(y, F)), y) & d(y, F) \neq 0 \\ (x, y) & d(y, F)=0 .\end{cases}
$$

Hence $K$ has FPP and so does $Q(T)$.

\section{KEY LEMMA}

Lemma 6. Let $X$ be a locally arcwise connected $B$-space and $Q(X)$ the same as in Theorem. Then $Q(X)$ has FPP.

Proof. Let $K$ be the set $\{\langle x, y\rangle \in Q(X) y \in F\}$. Then $Q(X) \backslash K$ is homeomorphic to $X \times(Y \backslash F)$. Let $\pi: Q(X) \backslash K \rightarrow X$ and $p: Q(X) \rightarrow Y$ be the projections defined by $\pi(\langle x, y\rangle)=x$ and $p(\langle x, y\rangle)=y$, respectively.

Now let $f: Q(X) \rightarrow Q(X)$ be any continuous mapping of $Q(X)$ into itself. On the contrary, suppose that $f$ has no fixed point. To lead to a contradiction, we shall divide our argument into four steps.

Step 1. We construct a sequence $\left\{z_{n}\right\}$ of points in $Q(X)$ and a nest $\left\{T_{n}\right\}$ of finite trees in $X$ satisfying the following four conditions. 
(3.1) $R_{n} f\left(z_{n}\right)=z_{n} \quad(n \geqq 1)$, where $R_{n}: Q(X) \rightarrow Q\left(T_{n-1}\right)$ is the retraction associated with the canonical retraction $r_{n}: X \rightarrow T_{n-1}$, and neither $z_{n}$ nor $f\left(z_{n}\right)$ belong to $K$.

(3.2) $\pi\left(z_{n}\right) \in T_{n-1}$ and $\pi f\left(z_{n}\right) \notin T_{n-1} \quad(n \geqq 1)$.

(3.3) $T_{n-1} \cap\left[\pi\left(z_{n}\right), \pi f\left(z_{n}\right)\right]=\left\{\pi\left(z_{n}\right)\right\}$.

(3.4) $T_{n}=\bigcup_{i=1}^{n}\left[\pi\left(z_{i}\right), \pi f\left(z_{i}\right)\right]$.

The sequences $\left\{z_{n}\right\}$ and $\left\{T_{n}\right\}$ are inductively obtained in the following manner.

Let $T_{0}$ be any point of $X$. Since $Q\left(T_{0}\right)$ is homeomorphic to $Y$, it has FPP. By Lemma 3, the restriction $R_{1} f \mid Q\left(T_{0}\right)$ is continuous. Therefore there exists a point $z_{1} \in Q\left(T_{0}\right)$ such that $R_{1} f\left(z_{1}\right)=z_{1}$. By our assumption that $f$ has no fixed point, neither $z_{1}$ nor $f\left(z_{1}\right)$ belong to $K$. Note that $\pi\left(z_{1}\right)=T_{0}$ and put $T_{1}=\left[\pi\left(z_{1}\right), \pi f\left(z_{1}\right)\right]$.

For positive integer $n$, since $Q\left(T_{n-1}\right)$ has FPP by Lemma 5 and $R_{n} f \mid Q\left(T_{n-1}\right)$ : $Q\left(T_{n-1}\right) \rightarrow Q\left(T_{n-1}\right)$ is continuous, there exists a point $z_{n} \in Q\left(T_{n-1}\right)$ such that $R_{n} f\left(z_{n}\right)=z_{n}$. Since $f$ has no fixed point by our assumption, neither $z_{n}$ nor $f\left(z_{n}\right)$ are in $K$.

Clearly $\pi\left(z_{n}\right) \in T_{n-1}$. However $\pi f\left(z_{n}\right) \notin T_{n-1}$. For if not, $f\left(z_{n}\right) \in$ $Q\left(T_{n-1}\right)$ and $R_{n} f\left(z_{n}\right)=f\left(z_{n}\right)$ because $R_{n}: Q(X) \rightarrow Q\left(T_{n-1}\right)$ is a retraction. Therefore $f\left(z_{n}\right)=z_{n}$, a contradiction. From (3.1) it follows that $r_{n} \pi f\left(z_{n}\right)=$ $\pi\left(z_{n}\right)$. Hence by the definition of $r_{n}$ we have $T_{n-1} \cap\left[\pi\left(z_{n}\right), \pi f\left(z_{n}\right)\right]=\pi\left(z_{n}\right)$. Put $T_{n}=T_{n-1} \cup\left[\pi\left(z_{n}\right), \pi f\left(z_{n}\right)\right]$.

Step 2. By our construction, the set $T=\bigcup_{n=1}^{\infty} T_{n}$ has the following four properties.

(3.5) For $n<m,\left[\pi\left(z_{n}\right), \pi f\left(z_{n}\right)\right] \cap\left[\pi\left(z_{m}\right), \pi f\left(z_{m}\right)\right]$ is empty or $\left\{\pi\left(z_{m}\right)\right\}$.

(3.6) If $e$ is an end point of $T$ then $e=\pi\left(z_{1}\right)$ or $e=\pi f\left(z_{n}\right)$ for some integer $n$.

(3.7) If $b$ is a branch point of $T$ then $b=\pi\left(z_{n}\right)$ for some integer $n$.

(3.8) If $b$ is a branch point of $T$ whose order is infinite, then there is an increasing sequence of integers $n_{1}<n_{2}<\cdots$ such that $\pi\left(z_{n_{i}}\right)=b$.

Step 3. If a subsequence $\left\{z_{n_{i}}\right\}$ of $\left\{z_{n}\right\}$ converges to a point $z$, then (3.9) and (3.10) hold.

(3.9) $p f(z)=p(z)$ and neither $z$ nor $f(z)$ are in $K$. For, $p f\left(z_{n_{i}}\right)=p\left(z_{n_{i}}\right)$ by (3.1) and hence $p f(z)=\lim p f\left(z_{n_{i}}\right)=\lim p\left(z_{n_{i}}\right)=p(z)$. Suppose, on the contrary, that $z \in K$. Then $p f(z)=p(z) \in F$. Therefore $f(z)=z$, a contradiction. Similarly $f(z)$ is not in $K$.

(3.10) There exists no arc $\alpha$ of $X$ such that $\pi\left(z_{n_{i}}\right) \in \alpha \cap T(i \geqq 1)$.

Suppose, on the contrary, that there exists such an arc $\alpha$. By (3.9) there exist $\pi(z)$ and $\pi f(z)$. Since $p f(z)=p(z)$ and $f(z) \neq z$, it holds that $\pi f(z) \neq \pi(z)$. Since $X$ is Hausdorff, there exist disjoint neighborhoods $U, V$ of $\pi f(z), \pi(z)$ in $X$, respectively. Let $U_{1}$ be a neighborhood of $\pi f(z)$ such that $U_{1} \subset U$ and each pair of points in $U_{1}$ can be joined by an arc in $U$. Since the map $\pi f$ is continuous, we can find a neighborhood $M$ of $\pi(z)$ in $\alpha$ and a neighborhood $N$ of $p(z)$ in $Y \backslash F$ such that $\pi f(M \times N) \subset U_{1}$. Let $z_{n_{k}}$, $z_{n_{l}}$ be in $M \times N$. Then $\pi\left(z_{n_{k}}\right), \pi\left(z_{n_{l}}\right)$ are in $M$ and $\pi f\left(z_{n_{k}}\right), \pi f\left(z_{n_{l}}\right)$ are distinct points in $\pi f(M \times N)$ by (3.2) and (3.3). Let $\beta$ be an arc in $U$ joining $\pi f\left(z_{n_{k}}\right)$ to $\pi f\left(z_{n_{l}}\right)$. The set $\left[\pi\left(z_{n_{k}}\right), \pi f\left(z_{n_{l}}\right)\right] \cup \beta \cup\left[\pi f\left(z_{n_{l}}\right), \pi\left(z_{n_{l}}\right)\right] \cup\left[\pi\left(z_{n_{l}}\right), \pi\left(z_{n_{l}}\right)\right]$ contains a simple closed curve in $X$, which contradicts Lemma 1 . 
Step 4. If the end points of $T$ are finite in number, by the construction of $T$ there exists a subsequence $\left\{z_{n_{1}}\right\}$ of $\left\{z_{n}\right\}$ such that $\left\{\left[\pi\left(z_{n_{1}}\right), \pi\left(z_{n_{i}}\right)\right], i>1\right\}$ is a nest of arcs. Let $\alpha$ be an arc of $X$ containing this nest. We may suppose that $\left\{\pi\left(z_{n_{i}}\right)\right\}$ and $\left\{p\left(z_{n_{i}}\right)\right\}$ converge to $a \in X$ and $y \in Y$, respectively. Put $(a, y)=z$. Then $\lim z_{n_{i}}=z$. This contradicts (3.10).

If the end points of $T$ are infinite in number, by Lemma 4 there exists a countable fan or a countable comb in $T$. For a countable fan with branch point $b$, by (3.8) there is an increasing sequence of integers $n_{1}<n_{2}<\cdots$ such that $\pi\left(z_{n_{i}}\right)=b$. We may suppose that $\left\{p\left(z_{n_{i}}\right)\right\}$ converges to a point $y \in Y$. Then $\lim z_{n_{i}}=(b, y)$. The point $b$ is contained in an arc of $X$, contrary to (3.10).

For a countable comb $C \subset T$, let $h: C_{0} \rightarrow C$ be a homeomorphism of $C_{0}$ onto $C$, where $C_{0}$ is the subset of $R^{2}$ in Definition 3. Let $a=h((0,0))$. By (3.7) we may put $\pi\left(z_{n_{k}}\right)=h((1 / k, 0))$ and assume that $\left\{p\left(z_{n_{k}}\right)\right\}$ converges to $y \in Y$. Then $\lim z_{n_{k}}=(a, y)$. The sequence $\left\{\pi\left(z_{n_{k}}\right)\right\}$ is contained in the arc $\alpha=\left[a, \pi\left(z_{n_{i}}\right)\right]$ of $X$, which violates (3.10).

\section{Proof of Theorem}

Definition 4. Let $S$ be a subset of an arcwise connected space. An arc component of $S$ is a maximal arcwise connected subset of $S$. Young's arc topology $[9,6]$ is the topology with the arc components of open sets of the given topology as basis.

Let $(X, \tau)$ be a $B$-space with topology $\tau$ and $Q(X, \tau)$ the quotient space in the theorem. Let $f: Q(X, \tau) \rightarrow Q(X, \tau)$ be any continuous mapping and $\lambda$ be Young's arc topology on $X$. Then $(X, \lambda)$ is a locally arcwise connected $T_{2}$-space. The mapping $f: Q(X, \lambda) \rightarrow Q(X, \tau)$ is continuous, because $\lambda$ is finer than $\tau$. Since $Q(X, \tau)$ is Hausdorff, the image of any arcwise connected subset under $f: Q(X, \lambda) \rightarrow Q(X, \tau)$ is arcwise connected. Hence the mapping $f: Q(X, \lambda) \rightarrow Q(X, \lambda)$ is continuous and has a fixed point by Lemma 6 . Thus $Q(X, \tau)$ has FPP.

\section{REFERENCES}

1. K. Borsuk, A theorem on fixed points, Bull. Acad. Polon. Sci. 2 (1954), 17-20.

2. _ Theory of retracts, Monograf. Mat. vol. 44, Warszawa, 1967.

3. W. Holsztyński, Fixed points of arcwise connected spaces, Fund. Math. 64 (1969), 289-312.

4. T. B. Muenzenberger and R. E. Smithon, The structure of nested spaces, Trans. Amer. Math. Soc. 201 (1975), 57-87.

5. V. P. Okhezin, Fixed-point theorems on products of spaces, Studies in Functional Analysis and its Applications, Ural. Gos. Univ., Sverdlovsk, 1985, pp. 72-80. (Russian)

6. R. E. Smithson, Changes of topology and fixed points for multi-valued functions, Proc. Amer. Math. Soc. 16 (1965), 448-454.

7. L. E. Ward, Jr., A fixed point theorem for chained spaces, Pacific J. Math. 9 (1959), 12731278.

8. G. T. Whyburn, Inward motions in connected spaces, Proc. Nat. Acad. Sci. U.S.A. 63 (1969), 271-274.

9. G. S. Young, Jr., The introduction of local connectivity by change of topology, Amer. J. Math. 68 (1946), 479-494. JAPAN

Faculty of Engineering, Kinki University, Hiro-Machi, Kure-shi, Hiroshima 737-01, 